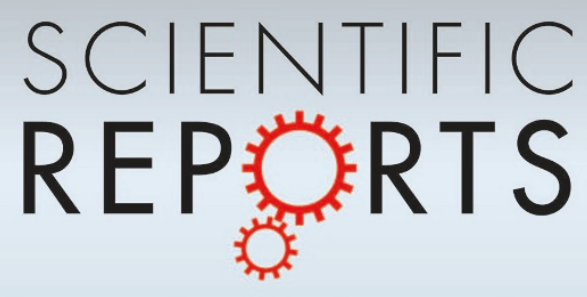

OPEN

SUBJECT AREAS:

BIOTECHNOLOGY

MOTOR CONTROL

PERIPHERAL NERVOUS SYSTEM

INHIBITION

Received

11 April 2013

Accepted

19 August 2013

Published

6 September 2013

Correspondence and requests for materials should be addressed to

E.D.J. (duco.jansen@ vanderbilt.edu)

\section{Transient and selective suppression of neural activity with infrared light}

\author{
Austin R. Duke', Michael W. Jenkins ${ }^{2}$, Hui Lu ${ }^{3}$, Jeffrey M. McManus ${ }^{3}$, Hillel J. Chiele ${ }^{3,2,4}$ \& E. Duco Jansen ${ }^{1,5}$
}

'Department of Biomedical Engineering, Vanderbilt University, Nashville, TN, USA, ${ }^{2}$ Department of Biomedical Engineering, Case Western Reserve University, Cleveland, OH, USA, ${ }^{3}$ Department of Biology, Case Western Reserve University, Cleveland, OH, USA, ${ }^{4}$ Department of Neurosciences, Case Western Reserve University, Cleveland, OH, USA, ${ }^{5}$ Department of Neurological Surgery, Vanderbilt University, Nashville, TN, USA.

Analysis and control of neural circuitry requires the ability to selectively activate or inhibit neurons. Previous work showed that infrared laser light selectively excited neural activity in endogenous unmyelinated and myelinated axons. However, inhibition of neuronal firing with infrared light was only observed in limited cases, is not well understood and was not precisely controlled. Using an experimentally tractable unmyelinated preparation for detailed investigation and a myelinated preparation for validation, we report that it is possible to selectively and transiently inhibit electrically-initiated axonal activation, as well as to both block or enhance the propagation of action potentials of specific motor neurons. Thus, in addition to previously shown excitation, we demonstrate an optical method of suppressing components of the nervous system with functional spatiotemporal precision. We believe this technique is well-suited for non-invasive investigations of diverse excitable tissues and may ultimately be applied for treating neurological disorders.

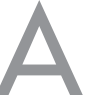

long with further elucidation of mechanisms underlying neural function, burgeoning interest in deep brain stimulation ${ }^{1}$, pain management ${ }^{2}$, functional electrical stimulation ${ }^{3}$, and brain-computer interfaces ${ }^{4}$ have created a demand for higher levels of neural specificity and control. Advances in electrical neural interface design have overcome many technical and biological challenges required to successfully excite and inhibit neural structures ${ }^{5,6}$. Additionally, novel optical methods of spatially and temporally precise neural control are increasing in popularity ${ }^{7,8}$. Optogenetics, a technique whereby light-responsive ion channels are introduced into targeted cells, is a promising new technology for exciting and inhibiting small groups of neurons with high spatial and temporal precision ${ }^{7}$. Recently, this technique was also used to inhibit motor nerve and muscle activity in transgenic mice? ${ }^{9}$. While an excellent tool for preclinical discovery, optogenetics is currently unavailable for clinical use due to the need for genetic manipulation.

In this work, we demonstrate the utility of an alternative optical technique for neural control using pulses of infrared light. The capability of pulsed infrared light to provide precise spatiotemporal activation of diverse excitable tissues has been shown ${ }^{10-18}$. In addition to neural excitation, we recently observed instances of suppressed activity in response to infrared light, though the mechanisms of action were unclear ${ }^{13,19}$. The response was reliable, repeatable, and occurred at a higher range of radiant energies than those used to induce excitation ${ }^{19}$. Recently, in the toadfish, it was demonstrated that spontaneous activity of sensory neurons innervating the vestibular system could be excited or inhibited by infrared light $t^{20}$.

An attractive attribute of thermal neuromodulation, such as infrared neural stimulation, is the ability to both activate and suppress neural activity. Infrared-induced activation is believed to be the result of a brief $(\sim \mathrm{ms})$ spatiotemporal gradient $(\mathrm{dT} / \mathrm{dt} \text { and } \mathrm{dT} / \mathrm{dx})^{15,18}$, whereas suppression is a function of an increase in baseline temperature $(\Delta \mathrm{T})$. By changing laser parameters (e.g. pulse width and frequency), one can either produce large, brief temperature transients for stimulation or relatively smaller temperature increases for suppression. While the discovery of controlled neural activation with infrared light was recently described, global temperature changes leading to block of nerve conduction, a phenomenon known as "heat block", has been investigated in both unmyelinated and myelinated nerves for decades ${ }^{21,22}$. Recent modeling studies also indicate the potential for block of action potential (AP) initiation and propagation with local increases in nerve temperature ${ }^{23}$.

Here we demonstrate the use of infrared pulses to transiently suppress electrically initiated AP generation and propagation, as well as neuromuscular function with high spatiotemporal specificity relative to currently available means of functional neural control. This effect was repeatedly demonstrated in both the unmyelinated buccal nerve 2 (BN2) of Aplysia californica, where it was used to inhibit specific sub-populations of the motor axons 
within the nerve, and the myelinated rat sciatic nerve. In this paper, we define "inhibition" as a transient elimination of action potential initiation, and "block" as a transient impediment to action potential propagation. The results of this study support the conclusion that the mechanism of infrared neural suppression, as with infrared stimulation, is thermally mediated.

\section{Results}

Inhibition of electrically initiated axonal activation in Aplysia. To investigate the inhibition of electrically initiated axonal activation, we first provided nonspecific supra-threshold electrical stimulation to the main trunk of BN2. Because the nerve was no longer attached to its ganglion (the buccal ganglion), it had no spontaneous activity. $\mathrm{BN} 2$ provides a robust and experimentally tractable ex vivo preparation with substantial length and a distal trifurcation allowing for simultaneous recording of multiple branches, and a muscular target that is known and tractable to study. Electrically evoked responses were recorded on the three distal branches of BN2: $\mathrm{BN} 2 \mathrm{a}, \mathrm{BN} 2 \mathrm{~b}$ and $\mathrm{BN} 2 \mathrm{c}^{24,25}$ - allowing the primary compound nerve action potential (CNAP) to be deconvolved and resolved into some of its spatial components. Two optical fibers flanked the pipette transverse to the nerve's longitudinal axis and were coupled to two independent diode (InGaAs) laser sources (Figure 1a). Laser 1 consisted of a tunable diode laser $\left(\lambda=1450 \mathrm{~nm}\right.$; $\left.\tau_{\text {po }}=0.5 \mathrm{~ms}\right)$ coupled to a $200 \mu \mathrm{m}$ diameter fiber optic. Laser 2 consisted of a similar diode laser $\left(\lambda=1860 \mathrm{~nm} ; \tau_{\text {po }}=5 \mathrm{~ms}\right)$ coupled to a $100 \mu \mathrm{m}$ diameter fiber optic. Differences in wavelengths and fiber optic diameters were not significant for the results we report (see Supplemental Discussion). Synchronizing the electrical stimulus with an infrared pulse from a single laser selectively eliminated an AP ordinarily appearing on one branch of BN2. Alternating between laser sources demonstrated that each laser eliminated different

a
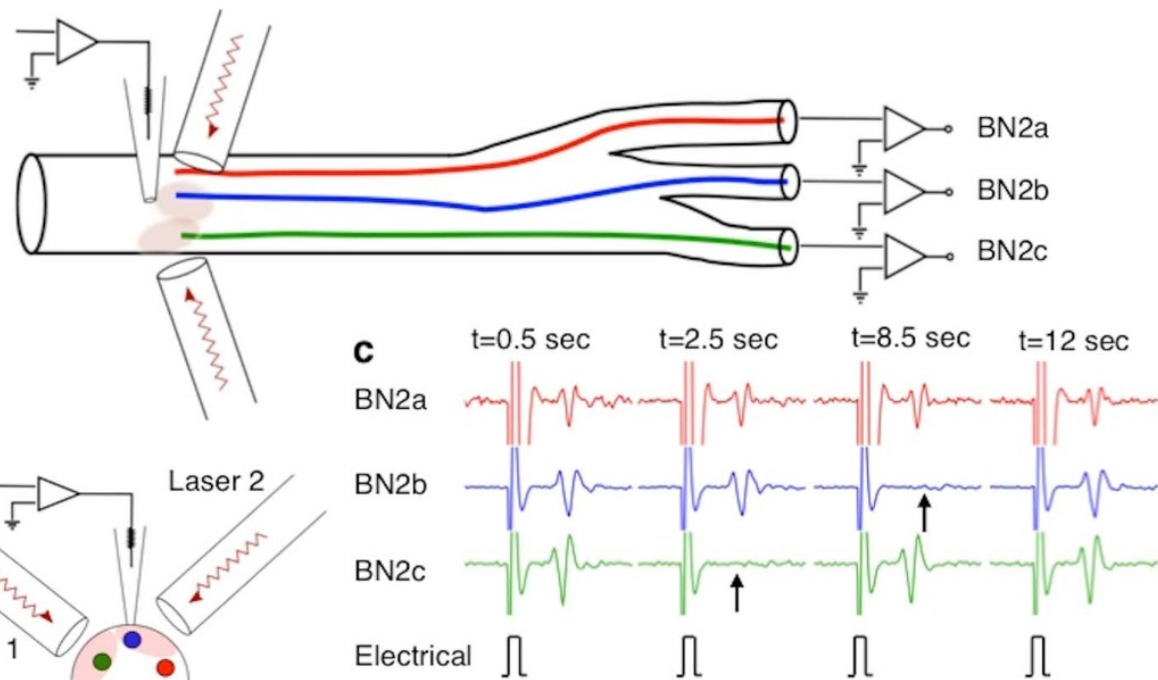

b

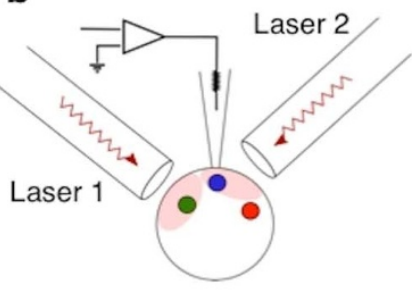

Laser 1

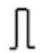

Laser 2

$\Omega$
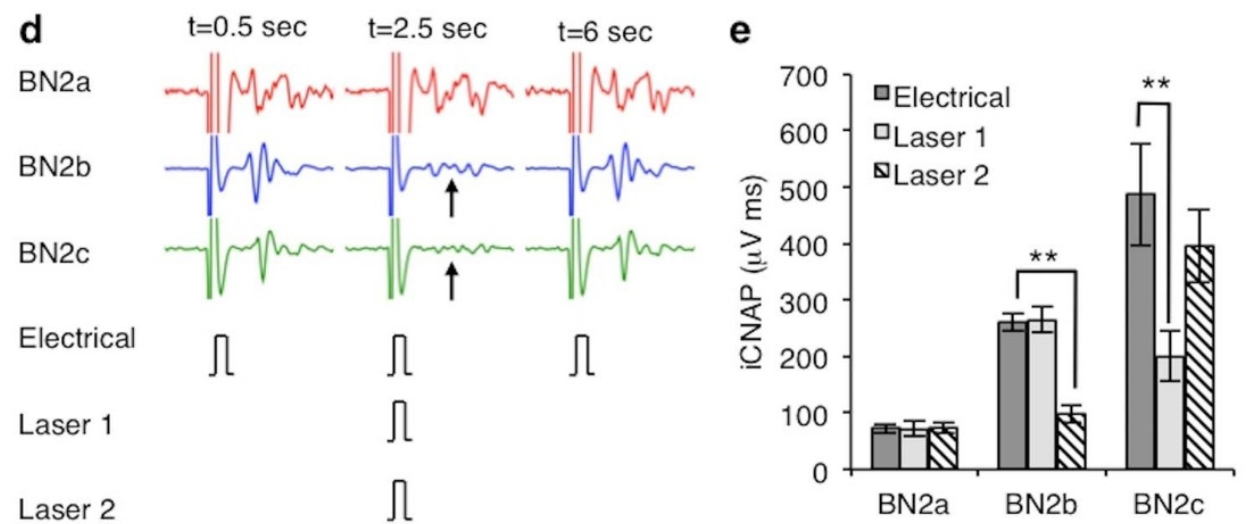

Figure 1 Infrared inhibition of AP initiation. (a) A micropipette providing supra-threshold extracellular electrical stimulation to BN2 is flanked by two optical fibers. Extracellular nerve recordings were obtained from the three distal branches. (b) Nerve cross-section schematic at the site of inhibition. (c) Neural recordings from branches of BN2 showing selective inhibition (arrows) of AP generation. Each laser inhibits initiation of an AP projecting to a single nerve branch. Laser $1\left(4.43 \pm 0.30 \mathrm{~J} / \mathrm{cm}^{2}\right)$ inhibits an AP projecting to BN2c, whereas Laser $2\left(8.34 \pm 0.78 \mathrm{~J} / \mathrm{cm}^{2}\right)$ inhibits an AP projecting to BN2b. Upon removal of the infrared pulse, electrically evoked APs return, indicating reversibility. (d) Neural recordings from branches of BN2 showing combined inhibition of two nerve branches. Applying infrared pulses from both lasers simultaneously inhibits APs projecting to BN2b and BN2c (arrows), while electrically evoked APs projecting to BN2a are unaffected. Each trace in (c) and (d) is 30 ms. (e) Average iCNAP recorded from BN2a, $\mathrm{BN} 2 \mathrm{~b}\left({ }^{* *} \mathrm{p}<0.01\right)$, and BN2c $(* * \mathrm{p}<0.01)$ in response to electrical only, electrical plus Laser 1 and electrical plus Laser $2(\mathrm{~N}=3 \mathrm{nerves} ; \mathrm{n}=5$ trials $)$. Data are presented as mean \pm SEM. 
electrically-evoked responses (Figure 1c). When both lasers provided pulses of infrared light simultaneously, responses on two branches were eliminated, while an electrically-activated response remained largely unchanged on the third branch (Figure 1d). Stopping the infrared pulses caused electrically evoked responses to reappear on all three branches, indicating that selective elimination of action potentials was completely reversible. The integrated CNAP (iCNAP) was used as a metric for the level of electrical activation for each branch. Each laser induced a reduction in the iCNAP magnitude of a selective axonal projection (Figure 1e). As a control, BN2a was not targeted; thus, no change in the iCNAP of BN2a was observed.

Infrared and electrical pulses were synchronized for the purpose of demonstrating temporally precise inhibition of action potential initiation. To further characterize infrared inhibition of action potential initiation, we investigated how the relative timing of electrical and infrared pulses affected threshold radiant exposures for inhibition. A single infrared pulse $\left(\lambda=1450 \mathrm{~nm}, \tau_{\mathrm{po}}=0.25 \mathrm{~ms}\right)$ was delivered over the range of $\mathrm{t}=-20 \mathrm{~ms}$ to $\mathrm{t}=0.5 \mathrm{~ms}$ relative to an electrical stimulus $\left(\tau_{\mathrm{pe}}=0.25 \mathrm{~ms}\right)$. For each trial, the electrical stimulus was $110 \%$ of the threshold current. We found that there existed a narrow window surrounding the delivery of the electrical pulse in which the infrared pulse can be applied to eliminate the electrically-initiated response. AP elimination reliably occurred with the infrared pulse delivered as much as $10 \mathrm{~ms}$ prior to the start of the electrical pulse. However, infrared pulses that were delivered earlier required radiant exposures that generated infrared stimulation in the absence of any electrical stimulus. Threshold radiant exposures for AP elimination rapidly increased when the infrared pulse was delivered after the electrical stimulus, primarily due to the evoked potential propagating away from the site of initiation before the infrared pulse arrived. AP elimination occurred reliably when the infrared pulse was delayed $0.25 \mathrm{~ms}$ after the electrical pulse, but was not observed when the infrared pulse was delayed by $0.5 \mathrm{~ms}$. Minimum threshold radiant exposures for AP elimination occurred when the infrared pulse was delivered $0.25 \mathrm{~ms}$ before the electrical pulse.

Nerve conduction block in Aplysia. We also demonstrated block of nerve conduction. A $200 \mu \mathrm{m}$ diameter fiber optic was positioned over the nerve trunk distal to the site of supra-threshold electrical stimulation $(\sim 1 \mathrm{~cm})$ targeting the proposed location of axons projecting to $\mathrm{BN} 2 \mathrm{c}$ (Figure 2a). A train of low radiant exposure $\left(0.50 \pm 0.02 \mathrm{~J} / \mathrm{cm}^{2}\right)$, high frequency $(200 \mathrm{~Hz})$ infrared pulses $(\lambda=$ $1450 \mathrm{~nm}, \tau_{\text {po }}=0.2 \mathrm{~ms}$ ) produced a rise in local tissue temperature and blocked responses projecting to BN2c (Figure 2b). During conduction block, BN2c iCNAP magnitude was lower than before and after the infrared train (Figure 2d). Blocked conduction began during the second half of the infrared pulse train and became stronger as the infrared block continued. This is likely due to the rate of temperature increase over the duration of the infrared train (Figure 2e). As infrared exposure begins, temperature may not be sufficient for block. However, as the temperature increases beyond the threshold for inhibition $\left(\sim 7.02^{\circ} \mathrm{C}\right)$, the effect is strengthened (Figure 2f). The induced temperature rise is discussed further in the Supplemental Materials. We also observed that BN2b experienced inhibition as well as enhancement in response to infrared exposure, and that this was consistent for a given nerve and fiber optic location (see Supplemental Fig. S1a-d and Supplemental Discussion).

Inhibition of neuromuscular contraction. To demonstrate functional relevance of infrared control, the effects on muscle force were measured. Distal BN2 muscle innervation was left intact and contraction force of the I1/I3 muscles was measured (Figure 3a). When infrared pulses $\left(\lambda=1450 \mathrm{~nm}, \tau_{\mathrm{po}}=0.2 \mathrm{~ms}, 0.50 \mathrm{~J} / \mathrm{cm}^{2}\right.$, $200 \mathrm{~Hz}$ ) were applied beginning 1 second before a propagating electrical stimulus, measured forces were reduced by nearly $80 \%$ when compared to control trials using only electrical stimulation
(Figure $3 b-d)$. Reduction in generated force was titrated by adjusting the radiant exposure of the infrared pulses (Supplemental Fig. S2). Results suggest that blocking part of the motor pool affects contraction in a specific muscle region (Supplemental Fig. S3 and Supplemental Movie S1).

Nerve conduction block in the rat sciatic nerve. Blocking and exciting effects of infrared exposure on propagating APs were validated in a myelinated mammalian nerve. Applying infrared pulses $(\lambda=$ $1450 \mathrm{~nm}, \tau_{\text {po }}=0.2 \mathrm{~ms}, 75.7 \pm 5.3 \mathrm{~mJ} / \mathrm{cm}^{2}, 200 \mathrm{~Hz}$ ) to the tibial branch of the rat sciatic nerve, approximately $1 \mathrm{~cm}$ distal to the site of electrical stimulation, reduced evoked EMG amplitude of the lateral gastrocnemius (LG) and the medial gastrocnemius (MG) (Figure 4). Enhanced propagated responses were observed as in Aplysia, were consistently repeatable, and depended on the location of the fiber optic and current path of the electrical stimulus (Supplemental Fig. S1e and Supplemental Discussion). Threshold temperature for reduction of $\mathrm{LG}$ integrated $\mathrm{EMG}(\mathrm{iEMG})\left(\sim 5.2^{\circ} \mathrm{C}\right)$ was determined as for Aplysia.

\section{Discussion}

Our results demonstrate the transient suppression of neural activity with infrared light, as well as potential utility of this technique. If the responses that we have reported are primarily due to temperature changes, they may have a variety of cellular and molecular effects. It should be noted, however, that in the context of the techniques currently available for manipulating neural activity, given the rapidity and the spatial selectivity with which the block we have described can be induced by infrared laser light, we believe that this new technique can be further optimized to serve as a precise, reversible form of non-contact neural control that avoids an electrical stimulation artifact. While the thermal effects on neural excitability are wellestablished, no previous study demonstrated this level of temporal and spatial precision. Previous demonstrations of complete thermal block required global changes to the temperature of the surrounding bath $^{26,27}$, whereas we are able to rapidly and reversibly alter excitability with spatial precision. In this study, transient suppression of neural excitability occurred within a spot as small as $100 \mu \mathrm{m}$ in diameter in as little as a few hundred $\mu$ s. We were limited by the combination of our laser output and size of optical fibers; thus, we could greatly reduce the population of neurons affected by using smaller fibers and free-space optical components to reduce the laser spot size.

Using this method of infrared laser-induced thermal block, electrically initiated responses were turned off in all Aplysia nerves tested (Supplemental Fig. S4) and functional selectivity (i.e. at the level of individual fascicles) was demonstrated (Figure 1). Although our preparation does not conclusively confirm inhibition of AP generation (rather than block of propagation after a very short distance), the selective inhibition of AP generation described by the models of Mou et al. suggest this is the case $\mathrm{e}^{23}$.

In addition to the likely inhibition of AP generation, we also demonstrated the ability to block AP propagation with functional selectivity. Providing a high frequency train $(200 \mathrm{~Hz})$ of lower radiant exposure pulses achieved specific and reproducible block of propagating APs in both Aplysia (Figure 2) and the rat (Figure 4) (see Supplemental Discussion). Mou et al. proposed that propagation block would require a greater local temperature increase distributed over a larger area than is required for initiation block ${ }^{23}$. This may be due to the AP safety factor allowing propagation to continue when local excitability is reduced ${ }^{28}$. While our protocol was aimed at producing a baseline temperature increase in a finite region of tissue, enhanced propagating responses observed in both Aplysia and rat nerves may be the result of sub-threshold electrical and thermal stimuli combining to achieve stimulation, as we have shown previously $^{19,29}$ (see Supplemental Discussion). Greater control over both excitation and inhibition will be achieved by optimizing light delivery 
a
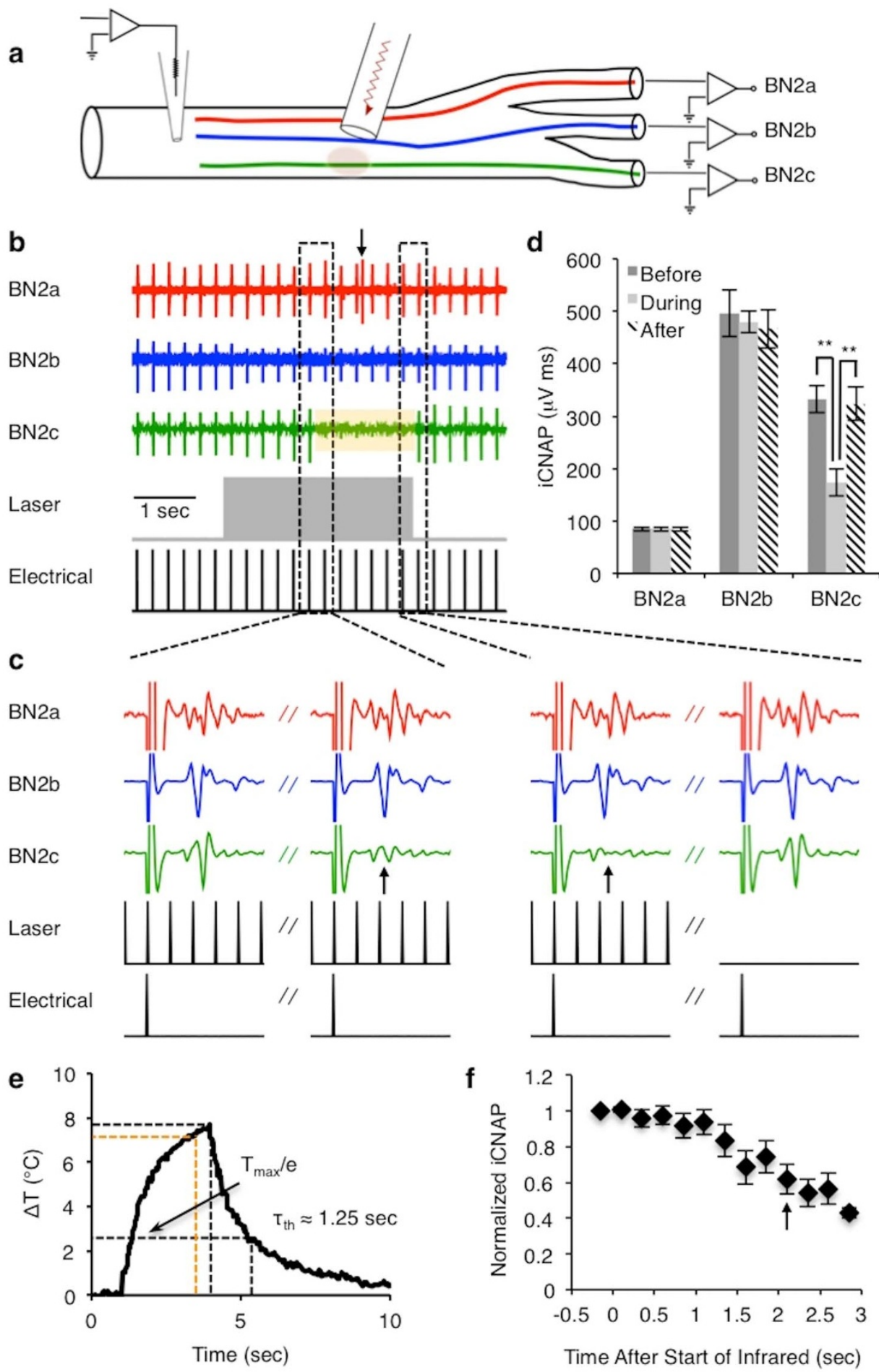

Figure $2 \mid$ Nerve conduction block in BN2 of Aplysia. (a) A micropipette electrically stimulated APs propagating to the three branches of BN2. Infrared pulses were delivered to the nerve via a $200 \mu \mathrm{m}$ diameter optical fiber, distal to the electrical stimulus. (b) Infrared exposure (indicated schematically by a gray bar) inhibits propagation of APs projecting to BN2c (highlighted by a yellow bar). A single spontaneous response is evident on the BN2a recording (arrow). This was occasionally observed before, during and after infrared exposure. APs on BN2b show some inhibition on this recording. Electrical artifacts are blanked for clarity. (c) Evoked and inhibited responses (arrows) are expanded. Each trace is 30 ms. (d) Average (1 sec) iCNAP for responses immediately preceding and following the infrared stimulus train, as well as the final $1 \mathrm{sec}$ of laser exposure. BN2c responses were selectively blocked during infrared inhibition ( ${ }^{* *} \mathrm{p}<0.01 ; \mathrm{N}=3$ nerves, $\mathrm{n}=11$ trials). (e) Local temperature increases by approximately $8^{\circ} \mathrm{C}$ during the 3 second infrared exposure. The thermal relaxation time is approximately $1.25 \mathrm{sec}$. (f) As duration of infrared exposure increased, magnitude of the BN2 iCNAP decreased. The location where reduction in BN2 iCNAP becomes significant $(\mathrm{p}<0.004)$ is indicated by an arrow in $(\mathrm{f})$ and orange dashed lines in $(e)$. Data are presented as mean \pm SEM. 
a

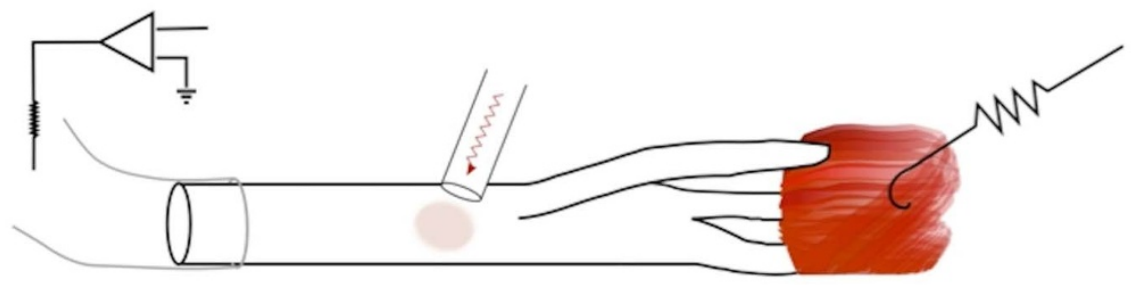

b

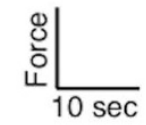

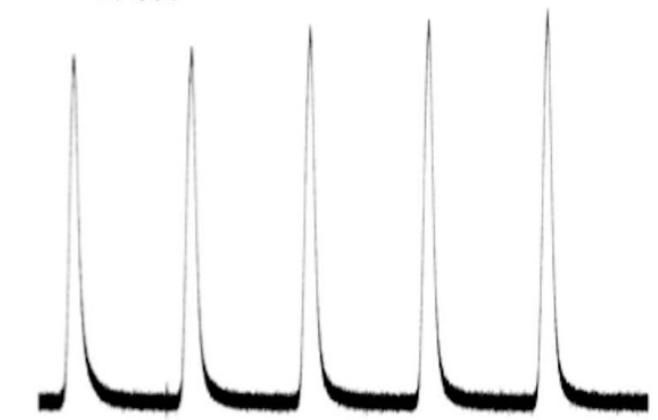

C

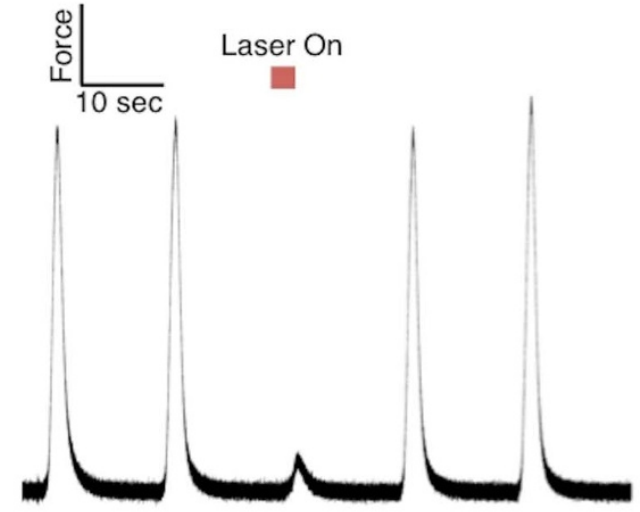

d

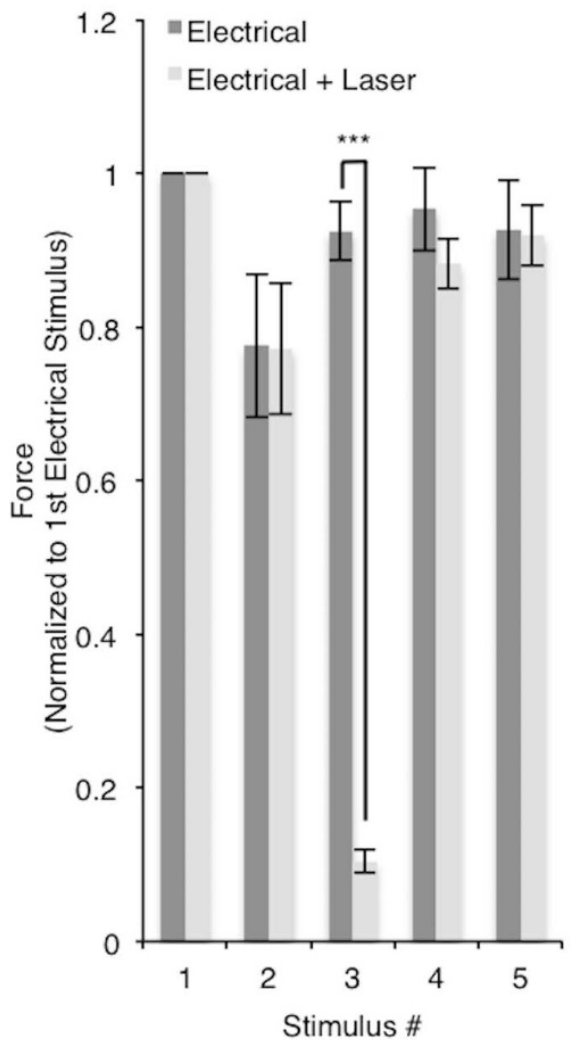

Figure 3 Infrared inhibition of electrically-evoked muscle contraction. (a) A suction electrode stimulated muscle contractions in the I1/I3 muscles as measured by a force transducer. A $200 \mu \mathrm{m}$ diameter optical fiber positioned distal $(\sim 1 \mathrm{~cm})$ to the electrical stimulus inhibited AP propagation of some motor units. (b) Electrically-evoked force in response to 2 -sec stimuli ( $\tau_{\mathrm{pe}}=1 \mathrm{~ms} ; 0.7 \mathrm{~mA} ; 10 \mathrm{~Hz}$ ) separated by a 12-second interval. (c) A 3-sec infrared pulse train $\left(\lambda=1450 \mathrm{~nm} ; \tau_{\mathrm{po}}=0.2 \mathrm{~ms} ; 0.54 \mathrm{~J} / \mathrm{cm}^{2}\right.$; indicated by horizontal red bar $)$ at $200 \mathrm{~Hz}$ delivered in conjunction with the third electrical stimulus inhibited force generation. (d) Average I1/I3 contraction force in response to electrical stimulation with and without the infrared pulse train (*** $\mathrm{p}<0.001$; without laser, $\mathrm{N}=2$ nerves, $\mathrm{n}=10$ trials; with laser, $\mathrm{N}=2$ nerves, $\mathrm{n}=10$ trials). Data are presented as mean $\pm \mathrm{SEM}$.

to the desired axons, for example by using smaller diameter fiber optics.

Given the results presented in this study, an explanation of how pulsed infrared light is capable of both excitation and suppression of neural activity may be suggested. It is important to note that the optical radiant exposures used in this study for suppression of neural activity are less than those required for infrared neural stimulation. As was shown previously, infrared neural stimulation is the result of a spatiotemporal temperature gradient ${ }^{18}$. The rapid temperature rise induces a transient change in the electrical capacitance of the neuron's plasma membrane, which in turn depolarizes the cell and induces a propagating $\mathrm{AP}^{15}$. Thus, simply raising the temperature will not induce the controlled, frequency-locked stimulation that may be accomplished with a brief, thermally-confined pulse. Similarly, many wavelengths are capable of eliciting excitation; the wavelengths used were chosen based on the desired penetration depth in neural tissue. Unlike infrared neural stimulation, local (and global) thermal neural inhibition is the result of non-uniform rate increases in the temperature-dependent Hodgkin-Huxley gating mechanisms, causing the rate of inactivation of sodium channels and activation of potassium channels to overwhelm activation of sodium channels ${ }^{22,23,30}$. This leads to either a faster and weaker response, or complete but reversible block of AP generation or propagation ${ }^{21,30}$.

The ultimate application of this technique will be contingent on the absence of long-term thermally-induced changes in tissue morphology or function. While infrared radiant exposures required for likely inhibition of AP generation are much lower than stimulation thresholds reported previously ${ }^{19}$, the maximum temperature rise induced during propagation block was approximately $8^{\circ} \mathrm{C}$ in Aplysia and $9^{\circ} \mathrm{C}$ in the rat (Figure 2e, Figure 4e and Supplemental Discussion). In Aplysia experiments reported here, responses were stable (i.e., no changes in threshold to induce action potential were observed) and no functional deficits (e.g. change in neural response or evoked force) or visibly identifiable damage was observed across multiple nerves during hours of intermittent stimulation. Further studies with molecular biological indicators are necessary to confirm 
a

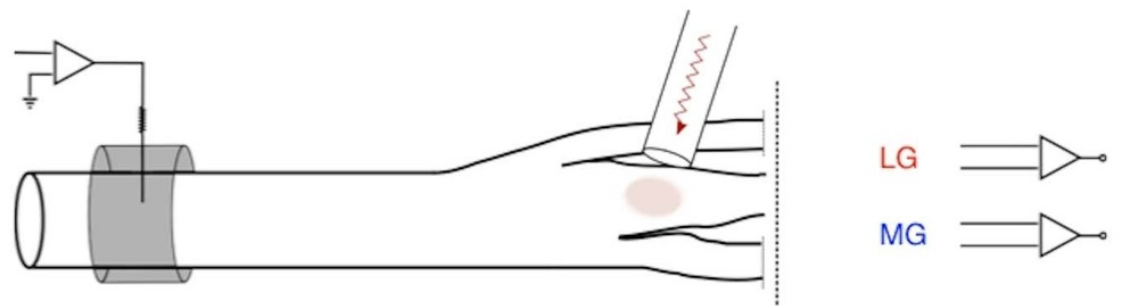

b

b

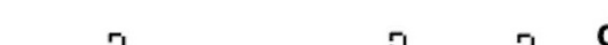

LG

MG

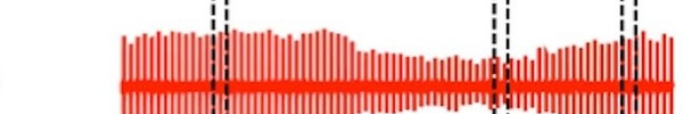

d

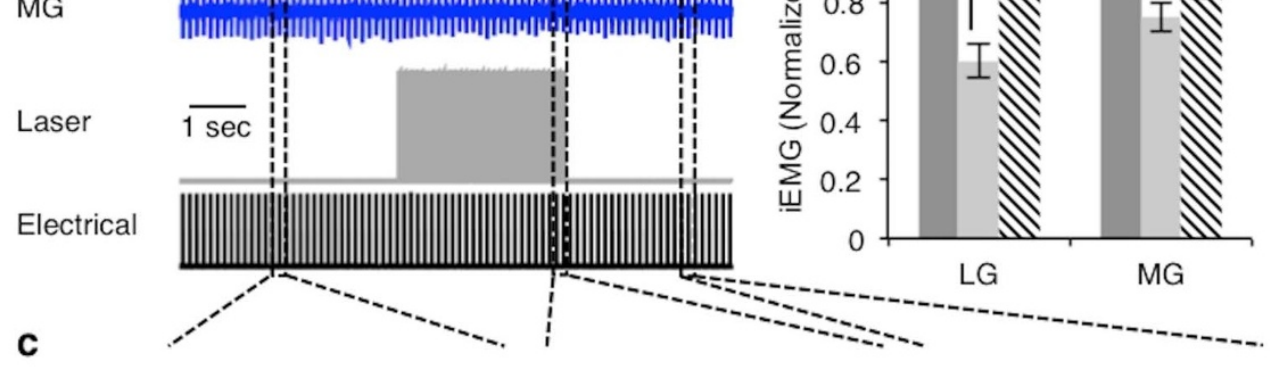

LG
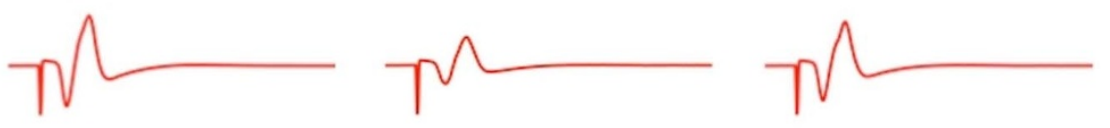

MG
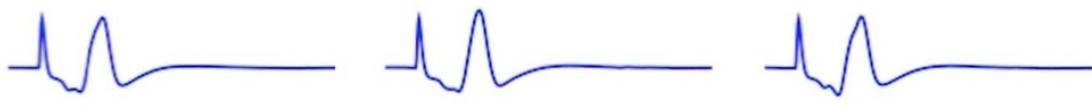

Laser
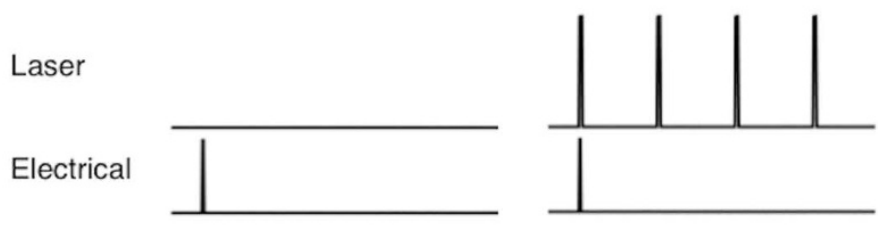

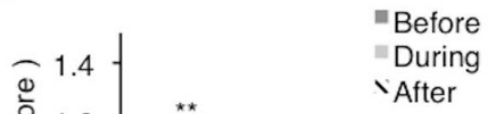

$\begin{array}{ll}\text { Ð }^{1.4} & \text { Before } \\ & \text { During } \\ & \text { vafter }\end{array}$
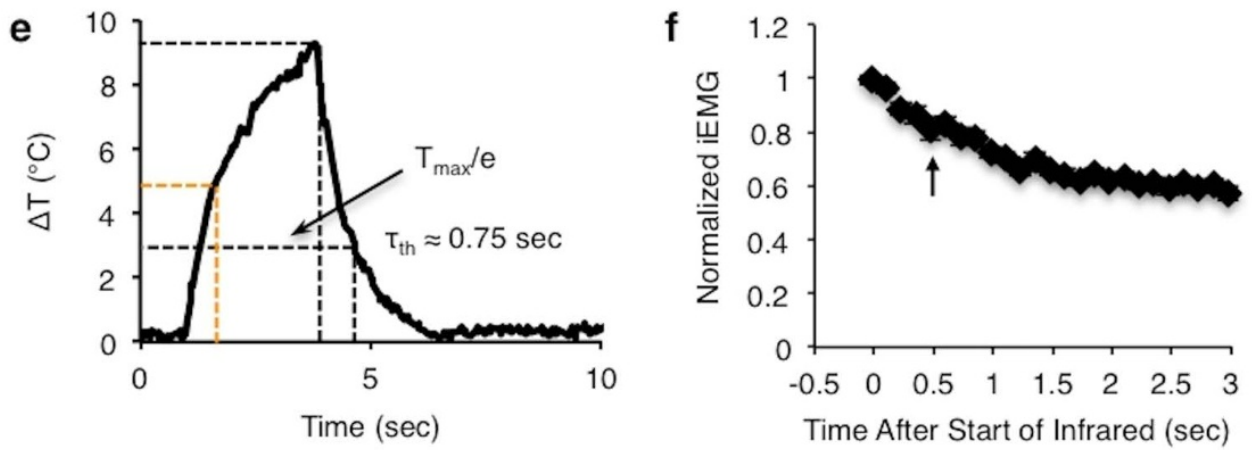

Figure $4 \mid$ Nerve conduction block in the rat sciatic nerve. (a) A monopolar cuff electrode stimulated propagating APs along the nerve trunk. A $400 \mu \mathrm{m}$ diameter fiber optic coupled to a diode laser was positioned over the tibial branch. (b) A train of infrared pulses $\left(\lambda=1450 \mathrm{~nm}\right.$; $\tau_{\text {po }}=0.2 \mathrm{~ms}$; indicated schematically by a gray bar) at $200 \mathrm{~Hz}$ reduces the amplitude of EMG recordings for MG and LG. Electrical artifacts are blanked for clarity. (c) Single EMG responses are shown before, during and after infrared inhibition. Each trace is $20 \mathrm{~ms}$. (d) Average iEMG for MG and LG before, during and after laser exposure ( ${ }^{* *} \mathrm{p}<0.01 ; \mathrm{N}=2$ nerves; $\mathrm{n}=12$ trials). Because of differences in absolute magnitude between the nerves tested, iEMG were normalized to the average iEMG for $1 \mathrm{sec}$ prior to infrared exposure. (e) Local temperature increased by approximately $9^{\circ} \mathrm{C}$ during the 3 second infrared exposure. The thermal relaxation time was approximately $0.75 \mathrm{sec}$. (f) Increasing duration of radiant exposure yielded greater reduction in the iEMG for LG. The location where reduction in iEMG for LG first becomes significant $(\mathrm{p}<0.002)$ is indicated by an arrow in $(\mathrm{f})$ and orange dashed lines in $(\mathrm{e})$. Data are presented as mean \pm SEM. 
the lack of thermally-induced tissue changes. Combining infrared and electrical pulses, similar to hybrid electro-optical stimulation ${ }^{19,29}$, may reduce requisite radiant exposures and alleviate concerns of damage.

Both unmyelinated and myelinated axons experience infrared suppression of neural activity, suggesting the mechanism of action between nerve fiber types is strongly related, and supporting the use of invertebrates for both fundamental and translational studies of the nervous system. Interestingly, non-excitable cells are also known to respond to infrared-induced spatiotemporal temperature gradients $^{15}$. The use of lasers to create highly localized thermal changes targeting specific cells or even localized regions of membrane may be a useful technique for probing cell function.

Infrared light is an intriguing tool for studying neural function and is being investigated for clinical applications ranging from cochlear implants $^{31}$ to intraoperative nerve monitoring ${ }^{32}$. Taken in combination with previous studies, including our own, exposure to infrared radiation is shown to be a multi-faceted phenomenon, capable of both exciting and suppressing neural activity. This points to a rich set of interactions between the nervous system and optical/thermal energy that may be exploited to further understand and control the nervous system and more general cell functions.

\section{Methods}

Aplysia preparation and nerve dissection. Aplysia californica $(\mathrm{n}=5)$ weighing 250 $350 \mathrm{~g}$ (Marinus Scientific, Long Beach, CA) were maintained in an aerated aquarium containing circulating artificial seawater (ASW) kept at $16-17^{\circ} \mathrm{C}$. The animals were fed dried seaweed every $1-3$ days.

Aplysia were anesthetized with an injection of $333 \mathrm{mM} \mathrm{MgCl}_{2}(\sim 50 \%$ of body weight) prior to dissection. Once anesthetized, animals were dissected and the buccal ganglia were removed and pinned in a recording dish and immersed in Aplysia saline (460 mM NaCl, $10 \mathrm{mM} \mathrm{KCl}, 22 \mathrm{mM} \mathrm{MgCl}_{2}, 33 \mathrm{mM} \mathrm{MgSO}_{4}, 10 \mathrm{mM} \mathrm{CaCl}_{2}, 10 \mathrm{mM}$ glucose, 10 mM HEPES, pH 7.6). Aplysia buccal ganglia are symmetric, so each hemiganglion has an associated buccal nerve 2 (BN2). Each BN2 was transected just distal to its attachment to its respective hemiganglion and anchored in place by pinning the protective sheath around the nerve to the Sylgard base (Dow Corning, Midland, MI) of the recording dish. Because the nerve was removed from its associated ganglion, little or no spontaneous neural activity was observed. A thermocouple was used to monitor the temperature of the bath, which was found to equilibrate to room temperature in a matter of minutes.

Aplysia electrophysiology. The three distal branches of BN2 were suctioned into nerve-recording electrodes to monitor the response to stimulation. Nerve-recording electrodes were made by hand-pulling polyethylene tubing ( $1.27 \mathrm{~mm}$ outer diameter, $0.86 \mathrm{~mm}$ inner diameter) over a flame to the desired inner diameter. Recording electrodes were suction-filled with Aplysia saline prior to suctioning of the nerve. Nerve signals were amplified $(\times 1000)$ and band-pass filtered $(300-500 \mathrm{~Hz})$ using an AC-coupled differential amplifier, digitized (Axon Digidata 1320A) and recorded (AxoGraph X)

Extracellular stimulating electrodes were made from thin-wall borosilicate capillary glass pulled to a diameter of about $40 \mu \mathrm{m}$ and resistances of about $0.1 \mathrm{M} \Omega$. For each experiment, an electrode was capillary-filled with Aplysia saline and positioned on the top surface of the nerve, in contact with the nerve sheath, using a micromanipulator. The return electrode was positioned at a distance in the bath to create monopolar stimulation. Monophasic currents supplied by a stimulus isolator were used for all experiments.

Delivery of infrared light to nerves. Two tunable diode laser systems were used throughout our investigation. Laser 1 consisted of a prototype tunable diode laser (Capella; Lockheed-Martin-Aculight, Bothell, WA) with wavelength $\lambda=1450 \mathrm{~nm}$ coupled to a $200 \mu \mathrm{m}$ diameter fiber optic (Ocean Optics, Dunedin, FL). Laser 2 comprised a similar and commercially available diode laser $(\lambda=1860 \mathrm{~nm})$ coupled to a $100 \mu \mathrm{m}$ diameter fiber optic. For rat experiments, Laser 1 was coupled to a $400 \mu \mathrm{m}$ diameter fiber optic. Fiber optics were secured in place using micromanipulators. The differences in laser wavelengths and fiber optic diameters were not critical for the results reported in this paper (see Supplemental Discussion).

Infrared inhibition of action potential generation in Aplysia. Fiber optics from laser systems 1 and 2 were positioned such that they flanked the stimulating electrode transverse to the nerve's longitudinal axis as shown in Figure 1a. Laser 1 supplied $0.5 \mathrm{~ms}$ pulses at $4.43 \pm 0.30 \mathrm{~J} / \mathrm{cm}^{2}$ and Laser 2 supplied $5 \mathrm{~ms}$ pulses at $8.34 \pm 0.78 \mathrm{~J} /$ $\mathrm{cm}^{2}$. Each trial $(\mathrm{N}=3$ nerves, $\mathrm{n}=5$ trials $)$ consisted of a series of repeating $500 \mathrm{~ms}$ episodes. For each episode, a monophasic electrical stimulus $\left(\tau_{\mathrm{pe}}=0.25 \mathrm{~ms}\right)$ providing current sufficient to generate consistent APs on all three recording electrodes $(461.4 \pm 36.2 \mu \mathrm{A})$ was applied at $\mathrm{t}=100 \mathrm{~ms}$. Because the laser pulses and the electrical pulse have different durations, they were all synchronized to end at the same time. This allowed total charge and total heat deposition to occur simultaneously. Effects of varying this time are discussed in the Supplemental Material. Each trial followed an ABACABACA pattern in which nerves were stimulated electrically (A), then either Laser 1 or Laser 2 was added (B), then the laser was removed leaving only electrical stimulation (A), followed by the other laser being added (C), and then the process was repeated. Nerve responses for each condition were analyzed using the integrated compound nerve action potential (iCNAP): the ensemble average for each condition within a given trial was rectified and summed over a finite interval following the electrical stimulation artifact.

Infrared inhibition of nerve conduction in Aplysia. The nerve preparation was as described previously, except a single $200 \mu \mathrm{m}$ fiber optic coupled to the $1450 \mathrm{~nm}$ laser source was positioned approximately $1 \mathrm{~cm}$ distal to the site of electrical stimulation, but proximal to the nerve trifurcation (Figure $2 \mathrm{a})$. Each trial $(\mathrm{N}=3$ nerves, $\mathrm{n}=11$ trials) consisted of one $10 \mathrm{sec}$ episode. Monophasic electrical stimuli $\left(\tau_{\mathrm{pe}}=0.25 \mathrm{~ms}\right.$; $659.1 \pm 18.9 \mu \mathrm{A})$ providing consistent responses on all three branches of BN2 were delivered at $4 \mathrm{~Hz}$ for the duration of the trial. At $4 \mathrm{sec}$, pulses of infrared light $\left(\tau_{\mathrm{po}}=\right.$ $0.2 \mathrm{~ms}$ ) were delivered at $200 \mathrm{~Hz}$ for 3 seconds. Nerve responses were analyzed using the iCNAP as described above.

Aplysia preparation for muscle force measurements. An Aplysia (422 g) was anesthetized as described above. The animal's buccal mass was removed and placed in a Petri dish containing a solution of $50 \%$ Aplysia saline and $50 \%$ isotonic $\mathrm{MgCl}_{2}$. Both buccal nerves 2 were severed at their attachment points to the buccal ganglia; removing the nerves from their associated ganglia ensured that there was little or no spontaneous activity. Incisions were made through the dorsal and ventral surfaces of the buccal mass, and further incisions were made to remove the radula-odontophore and pharyngeal tissue, leaving the I1/I3 muscle split into two separate halves with each half innervated by its buccal nerve 2 . The rest of the buccal mass and the ganglia were discarded. The muscle halves were moved to a recording dish with a Sylgard surface in the back half of the dish. Each I1/I3 half was glued by its anterior edge to the glass bottom of the dish just in front of the Sylgard. After gluing, the dish was filled with Aplysia saline. Each buccal nerve 2 was gently stretched and pinned on the Sylgard surface. Force transducers were attached to the medial portions of the I1/I3 halves using silk sutures.

Aplysia muscle force inhibition. Polyethylene suction electrodes were attached to the ends of each BN2. A $200 \mu \mathrm{m}$ diameter fiber optic coupled to the $1450 \mathrm{~nm}$ laser source was positioned distal $(\sim 1 \mathrm{~cm})$ to the suction electrode and proximal to the nerve trifurcation.

Electrical stimulation was applied using the nerve suction electrodes. Control trials consisted of 5 repetitions of electrical stimulation $\left(\tau_{\mathrm{pe}}=1 \mathrm{~ms}, 0.7 \mathrm{~mA}\right)$ delivered at $10 \mathrm{~Hz}$ for $2 \mathrm{sec}$. Each repetition was followed by an interval of 12 seconds with no stimulation (Figure 3b). Experimental trials consisted of the same protocol, but infrared pulses $\left(\tau_{\mathrm{po}}=0.2 \mathrm{~ms} ; 0.50 \mathrm{~J} / \mathrm{cm}^{2}\right)$ were applied at $200 \mathrm{~Hz}$ for 3 seconds beginning 1 second before the third electrical stimulus. Five control and experimental trials were repeated per parameter set with 3 min between each trial to allow the nerve to rest.

Movies of the muscle contraction were acquired for two of the experimental trials using a USB microscope positioned above the preparation.

Rat sciatic nerve preparation. All experiments were performed following protocols approved by the Vanderbilt University Institutional Animal Care and Use Committee (IACUC). Male Sprague-Dawley rats $(\mathrm{n}=2)$ weighing $250-300 \mathrm{~g}$ (Charles River) were anesthetized with continuously inhaled isoflurane (induction: $3 \%$ isoflurane, 3.0 LPM oxygen; maintenance: $2-2.5 \%$ isoflurane, 1.5 LPM oxygen). A rectal probe and heating pad were used to maintain the rat at a target body temperature of $35-37^{\circ} \mathrm{C}$ throughout the experiment. The animals were placed on a polycarbonate platform and their hindlimbs were shaved. The dorsal surface of the foot was then taped to the edge of the platform. An incision was made from the heel to the vertebral column and the skin was separated from the underlying tissue. The biceps femoris was then cut and divided proximal from the Achilles tendon to expose the sciatic nerve. The sural and peroneal branches of the sciatic nerve were transected so only innervation of the planterflexor muscles remained.

Rat sciatic nerve electrophysiology. Paired EMG electrodes made from perfluoroalkoxy (PFA)-coated silver wire (0.003" bare, 0.005 " coated) were inserted along the length of the medial gastrocnemius and lateral gastrocnemius muscles. EMG signals were amplified (x100) and band-pass filtered (100-1000 Hz) using an AC-coupled differential amplifier, digitized $(20 \mathrm{kHz}$; Axon Digidata 1440A) and recorded (AxoGraph X).

Infrared inhibition of nerve conduction in the rat sciatic nerve. A monopolar nerve cuff electrode was placed around the trunk of the sciatic nerve and a $400 \mu \mathrm{m}$ diameter fiber optic was positioned $500 \mu \mathrm{m}$ above the tibial branch. Each trial $(\mathrm{N}=3$ nerves, $\mathrm{n}$ $=15$ trials) consisted of one $10 \mathrm{sec}$ episode. Monophasic electrical stimuli ( $\tau_{\mathrm{pe}}=$ $0.1 \mathrm{~ms} ; 750 \mu \mathrm{A}$ ) were delivered at $4-8 \mathrm{~Hz}$ for the duration of the trial. At $4 \mathrm{sec}$, pulses of infrared light $\left(\tau_{\mathrm{po}}=0.2 \mathrm{~ms} ; 75.7 \mathrm{~mJ} / \mathrm{cm}^{2}\right)$ were delivered at $200 \mathrm{~Hz}$ for 3 seconds. Nerve responses were analyzed using the $\mathrm{iEMG}^{33}$, which was calculated in the same manner as the iCNAP described above. 
Data acquisition and analysis. Amplified and filtered nerve responses were acquired at $5 \mathrm{kHz}$. AxoGraph X software (AxoGraph Scientific) was used to coordinate stimulation and inhibition protocols, and to record acquired data. Post-acquisition data analysis was performed using a combination of AxoGraph X, Matlab (Matlab r2010b) and Microsoft Excel. The Kolmogorov-Smirnov test revealed that data were not normally distributed. Therefore, we compared data using the Mann-Whitney test with $\alpha=0.05$ and applied the Bonferroni method to adjust for multiple comparisons.

1. Martens, H. C. et al. Spatial steering of deep brain stimulation volumes using a novel lead design. Clin Neurophysiol 122, 558-566 (2011).

2. Slavin, K. V. Peripheral nerve stimulation for neuropathic pain. Neurotherapeutics 5, 100-106 (2008)

3. Peckham, P. H. \& Knutson, J. S. Functional electrical stimulation for neuromuscular applications. Annu Rev Biomed Eng 7, 327-360 (2005)

4. Konrad, P. \& Shanks, T. Implantable brain computer interface: challenges to neurotechnology translation. Neurobiol Dis 38, 369-375 (2010).

5. Grill, W. M., Norman, S. E. \& Bellamkonda, R. V. Implanted neural interfaces: biochallenges and engineered solutions. Annu Rev Biomed Eng 11, 1-24 (2009).

6. Kilgore, K. L. \& Bhadra, N. Nerve conduction block utilising high-frequency alternating current. Med Biol Eng Comput 42, 394-406 (2004).

7. Fenno, L., Yizhar, O. \& Deisseroth, K. The development and application of optogenetics. Annu Rev Neurosci 34, 389-412 (2011).

8. Richter, C. P., Matic, A. I., Wells, J. D., Jansen, E. D. \& Walsh, J. T. Neural stimulation with optical radiation. Laser Photonics Rev 5, 68-80 (2011).

9. Liske, H. et al. Optical inhibition of motor nerve and muscle activity in vivo. Muscle Nerve 47, 916-921 (2013)

10. Fried, N. M., Lagoda, G. A., Scott, N. J., Su, L. M. \& Burnett, A. L. Noncontact stimulation of the cavernous nerves in the rat prostate using a tunable-wavelength thulium fiber laser. J Endourol 22, 409-413 (2008).

11. Izzo, A. D., Richter, C. P., Jansen, E. D. \& Walsh, J. T., Jr. Laser stimulation of the auditory nerve. Lasers Surg Med 38, 745-753 (2006).

12. Jenkins, M. W. et al. Optical pacing of the embryonic heart. Nat Photonics 4 623-626 (2010).

13. Cayce, J. M., Friedman, R. M., Jansen, E. D., Mahavaden-Jansen, A. \& Roe, A. W. Pulsed infrared light alters neural activity in rat somatosensory cortex in vivo. Neuroimage 57, 155-166 (2011).

14. Dittami, G. M., Rajguru, S. M., Lasher, R. A., Hitchcock, R. W. \& Rabbitt, R. D. Intracellular calcium transients evoked by pulsed infrared radiation in neonatal cardiomyocytes. J Physiol 589, 1295-1306 (2011).

15. Shapiro, M. G., Homma, K., Villarreal, S., Richter, C. P. \& Bezanilla, F. Infrared light excites cells by changing their electrical capacitance. Nat Commun 3, 736 (2012).

16. Teudt, I. U., Nevel, A. E., Izzo, A. D., Walsh, J. T., Jr. \& Richter, C. P. Optical stimulation of the facial nerve: a new monitoring technique? Laryngoscope 117, 1641-1647 (2007)

17. Wells, J., Kao, C., Jansen, E. D., Konrad, P. \& Mahadevan-Jansen, A. Application of infrared light for in vivo neural stimulation. J Biomed Opt 10, 064003 (2005).

18. Wells, J. et al. Biophysical mechanisms of transient optical stimulation of peripheral nerve. Biophys J 93, 2567-2580 (2007).

19. Duke, A. R., Lu, H., Jenkins, M. W., Chiel, H. J. \& Jansen, E. D. Spatial and temporal variability in response to hybrid electro-optical stimulation. J Neural Eng 9, 036003 (2012)

20. Rajguru, S. M. et al. Infrared photostimulation of the crista ampullaris. J Physio 589, 1283-1294 (2011).

21. Hodgkin, A. L. \& Katz, B. The effect of temperature on the electrical activity of the giant axon of the squid. J Physiol 109, 240-249 (1949).

22. Rattay, F. \& Aberham, M. Modeling axon membranes for functional electrical stimulation. IEEE Trans Biomed Eng 40, 1201-1209 (1993).

23. Mou, Z., Triantis, I. F., Woods, V. M., Toumazou, C. \& Nikolic, K. A simulation study of the combined thermoelectric extracellular stimulation of the sciatic nerve of the Xenopus laevis: the localized transient heat block. IEEE Trans Biomed Eng 59, 1758-1769 (2012).

24. Warman, E. N. \& Chiel, H. J. A new technique for chronic single-unit extracellular recording in freely behaving animals using pipette electrodes. J Neurosci Methods 57, 161-169 (1995).

25. Nargeot, R., Baxter, D. A. \& Byrne, J. H. Contingent-dependent enhancement of rhythmic motor patterns: an in vitro analog of operant conditioning. J Neurosci 17, 8093-8105 (1997).

26. Davis, F. A., Schauf, C. L., Reed, B. J. \& Kesler, R. L. Experimental studies of the effects of extrinsic factors on conduction in normal and demyelinated nerve. 1. Temperature. J Neurol Neurosurg Psychiatry 39, 442-448 (1976).

27. Xu, D. \& Pollock, M. Experimental nerve thermal injury. Brain 117(Pt 2), 375-384 (1994)

28. Hodgkin, A. L. Evidence for electrical transmission in nerve: Part I. J Physiol 90, 183-210 (1937)

29. Duke, A. R. et al. Combined optical and electrical stimulation of neural tissue in vivo. J Biomed Opt 14, 060501 (2009).

30. Huxley, A. F. Ion movements during nerve activity. Ann N Y Acad Sci 81, 221-246 (1959).

31. Rajguru, S. M. et al. Optical cochlear implants: evaluation of surgical approach and laser parameters in cats. Hear Res 269, 102-111 (2010).

32. Fried, N. M. et al. Identification and Imaging of the Nerves Responsible for Erectile Function in Rat Prostate, In Vivo, Using Optical Nerve Stimulation and Optical Coherence Tomography. Selected Topics in Quantum Electronics, IEEE Journal of 13, 1641-1645 (2007).

33. Llewellyn, M. E., Thompson, K. R., Deisseroth, K. \& Delp, S. L. Orderly recruitment of motor units under optical control in vivo. Nat Med 16, 1161-1165 (2010).

\section{Acknowledgements}

This work was made possible by funds from the Department of Defense (HR0011-10-1-0074), the National Institutes of Health (NS-047073) and the National Science Foundation (DMS-1010434). We would like to thank Dr. Dustin Tyler and three anonymous reviewers for their helpful suggestions and careful critiques that improved this manuscript.

\section{Author contributions}

A.R.D. designed and performed all experiments, analyzed the data and wrote the manuscript. M.W.J. and H.J.C. designed all experiments, performed Aplysia experiments and analyzed data. H.L. and J.M.M. performed Aplysia experiments. E.D.J. designed experiments. All authors reviewed and edited the manuscript, as well as participated in discussing the results and their implications.

\section{Additional information}

Supplementary information accompanies this paper at http://www.nature.com/ scientificreports

Competing financial interests: Some of the authors (ARD and EDJ) are inventors on intellectual property in the subject area of the paper. This IP is owned by the inventors' institution (Vanderbilt University) and is or may be licensed to a third party (Lockheed-Martin). Royalty distributions are made according to standard university policy. How to cite this article: Duke, A.R. et al. Transient and selective suppression of neural activity with infrared light. Sci. Rep. 3, 2600; DOI:10.1038/srep02600 (2013).

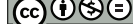

This work is licensed under a Creative Commons AttributionNonCommercial-NoDerivs 3.0 Unported license. To view a copy of this license visit http://creativecommons.org/licenses/by-nc-nd/3.0 